\title{
ALMOST COMPLEX TORIC MANIFOLDS AND POSITIVE LINE BUNDLES
}

\author{
AKIO HATTORI \\ Department of Mathematics, Meiji University \\ 1-1-1 Higashimita, Tama-ku, Kawasaki-shi, 214 Japan \\ E-mail: hattori@math.meiji.ac.jp
}

1. Introduction. Masuda $[\mathrm{M}]$ developed the theory of unitary toric manifolds which generalized the theory of toric varieties and the theory of Hamiltonian toral manifolds (toric manifolds) due to Delzant [D] in some direction. There are works of Karshon and Tolman $[\mathrm{KT}]$ and of Grossberg and Karshon [GK] in a similar direction. An advantage of Masuda's theory is the introduction of the notion of multi-fan attached to unitary toric manifolds. The multi-fan is essentially a simplicial complex with some extra data and equipped with a map from the set of its vertices to the second homology group $H_{2}(B T ; \mathbf{Z})$ of the classifying space $B T$ of the torus $T$ acting on the manifold.

One of the main results of $[\mathrm{M}]$ is the multiplicity formula which relates the index of the Dirac operator twisted by a line bundle $L$ acted on by the torus $T$ to some data coming from the moment map of the bundle. Similar formulas are also in [KT] and [GK]. The moment map is a $T$-invariant map from $M$ into the second cohomology with real coefficients $H^{2}(B T, \mathbf{R})$. Irreducible representations of a torus $T$ are one dimensional. They form an abelian multiplicative group $\operatorname{Hom}\left(T, S^{1}\right)$. We identify $\operatorname{Hom}\left(T, S^{1}\right)$ with $H^{2}(B T, \mathbf{Z})$. So, if $\chi$ is a virtual representation of $T$ then it can be written as

$$
\chi=\sum_{u \in H^{2}(B T, \mathbf{Z})} m(u) \chi^{u}, m(u) \in \mathbf{Z},
$$

where $\chi^{u}$ is the irreducible representation corresponding to $u$. The multiplicity formula identifies the integer $m(u)$ with the value $d_{L}^{\prime}(u)$ of a degree function $d_{L}^{\prime}$ defined on $H^{2}(B T, \mathbf{R})$ minus a union of certain affine hyperplanes when $\chi$ is the index of the Dirac operator $D_{L}$ twisted by the line bundle $L$.

In this paper we shall deal with almost complex toric manifolds satisfying some mild conditions. Given a $T$-line bundle we define a piecewise linear map $\Psi_{L}$ from the realization of the first barycentric subdivision of the simplicial complex attached to the multi-fan

1991 Mathematics Subject Classification: 57S25, 58F05.

The paper is in final form and no version of it will be published elsewhere. 
into $H^{2}(B T, \mathbf{R})$ such that its image is contained in the union of hyperplanes described above. Theorem 3.5, work done jointly with Masuda, states that the winding number $\bar{d}_{L}(u)$ of the map $\Psi_{L}$ around $u \in H^{2}(B T, \mathbf{R})$ coincides with the value $d_{L}(u)$ of the degree function $d_{L}$ which is intimately related to $d_{L}^{\prime}$. The same sort of statement (Theorem 3.11) holds also for the degree function of [KT]. This allows us to express the multiplicity $m(u)$ purely in terms of the multi-fan and algebraic data of the line bundle $L$.

We shall also define a notion of positiveness of line bundles in such a way that analogues of the Nakai criterion and the Kodaira vanishing theorem hold (Corollary 4.8 and Theorem 4.21; also see Theorem 4.19). Furthermore, it is shown (Theorem 5.4) that if the Todd genus of the manifold is equal to 1 , then the above definition of positiveness is quite parallel to the classical convexity criterion of ample line bundles in the theory of toric varieties, cf. e.g. [F],[O].

The organization of the paper is as follows. In Section 2 we review Masuda's theory in a way suitable to our purpose. Section 3 is devoted to the results surrounding the map $\Psi$. In Section 4 we introduce the notion of positiveness of line bundles and give proof of the analogues of the Nakai criterion and the Kodaira vanishing theorem. In proving the Nakai criterion a formula in Theorem 4.2 which expresses the number $c_{1}(L)^{n}[M]$ in terms of the degree function $\bar{d}_{L}$ is crucial. For the proof of Theorem 4.2 we use a combinatorial formula (Lemma 4.5) concerning the volume of rational polytopes. The formula is a simple one but seems to be new. In the last section, Section 5 , the case where the Todd genus equals 1 will be dealt with.

The author would like to thank M. Masuda for stimulating conversations and cooperation.

2. Almost complex toric manifolds and multi-fan. A closed, connected 2ndimensional almost complex manifold $M$ acted on by a torus $T$ will be called an almost complex toric manifold if the following conditions are satisfied.

1. The action preserves the almost complex structure.

2. If $T_{0}$ denotes the trivializer of the action, then $\operatorname{dim} T / T_{0}=n$.

3. The fixed point set of the action (which we denote by $M^{T}$ ) is not empty.

We set $\bar{T}=T / T_{0} . \bar{T}$ acts effectively on $M$, and $M^{T}$ is an isolated set.

According to $[\mathrm{M}]$ a closed, connected codimension 2 submanifold $M_{i}$ of $M$ will be called a characteristic submanifold if it is a fixed point set component of a certain subcircle $\bar{S}_{i}$ of $\bar{T}$ and $M_{i} \cap M^{\bar{T}} \neq \emptyset . M_{i}$ inherits the almost complex structure from $M$. Let $\Sigma_{M}^{0}$ denote the set of all indices $i$ of characteristic submanifolds $M_{i}$. We set

$$
\Sigma_{M}^{k-1}=\left\{I=\left\{i_{1}, i_{2}, \ldots i_{k}\right\} ; M_{I}=M_{i_{1}} \cap \cdots \cap M_{i_{k}} \neq \emptyset, i_{\nu} \in \Sigma_{M}^{0}\right\} .
$$

Then $\Sigma_{M}^{0}, \Sigma_{M}^{1}, \ldots, \Sigma_{M}^{n-1}$ form a simplicial set $\Sigma_{M}$ (in [M] this simplicial set was denoted by $\left.\Gamma_{M}\right)$. Note that all $M_{I}$ are also almost complex toric manifolds. In the sequel we shall make the following assumption.

$$
\text { All } M_{I} \text { are connected and } M_{I} \cap M^{\bar{T}} \neq \emptyset \text {. }
$$

The assumption implies in particular $M_{I}$ is a point of $M^{T}$ for any $(n-1)$-simplex $I \in \Sigma_{M}^{n-1}$. 
Let $\nu_{i}$ be the normal bundle of $M_{i}$ in $M$. It is a complex line bundle. Denoting by $f_{i}: M_{i} \rightarrow M$ the inclusion map we define $\xi_{i} \in H_{\bar{T}}^{2}(M ; \mathbf{Z})$ by $\xi_{i}=f_{i *}(1)$ where $f_{i *}: H_{\bar{T}}^{0}(M ; \mathbf{Z}) \rightarrow H_{\bar{T}}^{2}(M ; \mathbf{Z})$ is the Gysin homomorphism of $f_{i}$. If $p \in M_{i} \cap M^{T}$, then $\nu_{i} \mid p$ is an irreducible $\bar{T}$ module, that is, $\nu_{i} \mid p \in \operatorname{Hom}\left(\bar{T}, S^{1}\right)$. The restriction of $\nu_{i} \mid p$ to $\bar{S}_{i}$ does not depend on the choice of $p$ in $M_{i} \cap M^{T}$.

$\operatorname{Hom}\left(\bar{T}, S^{1}\right)$ is an abelian multiplicative group. We shall identify it with $H^{2}(B \bar{T} ; \mathbf{Z})$ by the isomorphism $\operatorname{Hom}\left(\bar{T}, S^{1}\right) \rightarrow H^{2}(B \bar{T} ; \mathbf{Z})$ given by $\alpha \mapsto c_{1}^{\bar{T}}(\alpha)$, where $c_{1}^{\bar{T}}$ is the equivariant first Chern class. The $\bar{T}$-module corresponding to $u \in H^{2}(B \bar{T} ; \mathbf{Z})$ is denoted by $\chi^{u}$. For example, $\nu_{i} \mid p=\chi^{\xi_{i} \mid p}$.

Lemma $2.1\left([\mathrm{M}]\right.$, Lemma 1.3). Take $I \in \Sigma_{M}^{n-1}$ and $p \in M_{I}$. Then the set $\left\{\xi_{i} \mid p, i \in I\right\}$ forms a basis of $H^{2}(B \bar{T} ; \mathbf{Z})$. In particular, $\xi_{i} \mid p$ is a primitive element.

In a similar way there is a standard isomorphism between $\operatorname{Hom}\left(S^{1}, \bar{T}\right)$ and $H_{2}(B \bar{T} ; \mathbf{Z})$. The embedding $\bar{S}_{i} \hookrightarrow \bar{T}$ determines a primitive element of $H_{2}(B \bar{T} ; \mathbf{Z})$ up to sign and hence a primitive element $v_{i} \in H_{2}(B \bar{T} ; \mathbf{Z})$ up to sign. The sign will be determined by requiring

$$
<\xi_{i} \mid p, v_{i}>=1
$$

where $<>$ is the coupling between cohomology and homology. It follows easily that $\left\{v_{i} ; i \in I\right\}$ is the dual basis of $\left\{\xi_{i} \mid p, i \in I\right\}$. The following lemma will play an important role in the sequel.

Lemma $2.2([\mathrm{M}]$, Lemma 1.5). There is an identity

$$
u=\sum_{i \in \Sigma_{M}^{0}}<u, v_{i}>\xi_{i} \in \hat{H}_{\bar{T}}^{2}(M ; \mathbf{R})
$$

which holds for any $u \in H^{2}(B \bar{T} ; \mathbf{R})$. Here $\hat{H}_{\bar{T}}^{2}(M ; \mathbf{R})$ is the degree 2 part of $H_{\bar{T}}^{*}(M ; \mathbf{R}) / S$ torsion where $S$ is the multiplicative set generated by non-zero elements in $H^{2}(B \bar{T} ; \mathbf{R})$.

The simplicial set $\Sigma_{M}$ is equipped with a projection map $\pi: \Sigma_{M}^{0} \rightarrow H_{2}(B \bar{T} ; \mathbf{Z})$ defined by $\pi(i)=v_{i}$. It induces a piecewise affine map $\pi:\left|\Sigma_{M}\right| \rightarrow H_{2}(B \bar{T} ; \mathbf{R})$, where $\left|\Sigma_{M}\right|$ is the realization of $\Sigma_{M}$. We shall denote by $s_{I}$ the realization of $I \in \Sigma_{M}$ in $\left|\Sigma_{M}\right|$. For each $I, \pi$ maps $s_{I}$ injectively on the affine simplex $s_{I}^{\prime}$ in $H_{2}(B \bar{T} ; \mathbf{R})$ spanned by $\left\{v_{i} ; i \in I\right\}$. Once an orientation of $H_{2}(B \bar{T} ; \mathbf{R})$ is fixed, each $(n-1)$-simplex $s_{I}^{\prime}\left(I \in \Sigma_{M}^{n-1}\right)$ will be given the orientation $o_{I}$ defined in the following way. Fix $i \in I$. The vector $v_{i}$ intersects $s_{I}^{\prime}$ transversally. Requiring the positive orientation of the vector $v_{i}$ followed by $o_{I}$ should coincide with the given orientation of $H_{2}(B \bar{T} ; \mathbf{R})$ determines $o_{I}$. This does not depend on the choice of $i \in I$.

LEMma 2.3. $\left|\Sigma_{M}\right|$ is a closed pseudo-manifold. This means that every simplex is contained in some $(n-1)$-dimensional simplex, and, for each $J \in \Sigma_{M}^{n-2}, s_{J}$ is the face of precisely two $(n-1)$-simplices $s_{I}$ and $s_{I^{\prime}}$. Moreover, if an orientation of $H_{2}(B \bar{T} ; \mathbf{R})$ is fixed and each $(n-1)$-simplex $s_{I}$ is oriented so that $\pi \mid s_{I}: s_{I} \rightarrow s_{I}^{\prime}$ preserves the orientation, then $\sum s_{I}$ is the fundamental class of $\left|\Sigma_{M}\right|$. This means that, if $\left|\Sigma_{M}\right|=\bigcup_{\nu}\left|\Sigma_{M}\right|_{\nu}$ is the decomposition into connected component of $\left|\Sigma_{M}\right|$, then $\sum_{s_{I} \subset\left|\Sigma_{M}\right|_{\nu}} s_{I}$ generates $H_{n-1}\left(\left|\Sigma_{M}\right|_{\nu} ; \mathbf{Z}\right)$ for each $\nu$. 
Proof. Take $J \in \Sigma_{M}^{n-2}$. By virtue of (2.1) $M_{J}$ is a connected almost complex submanifold of dimension 2 on which the torus $T$ acts non-trivially so that it is complex projective line and has precisely two fixed points $M_{I}$ and $M_{I^{\prime}}$. This means that $I$ and $I^{\prime}$ are only simplices to which $J$ is incident, namely $s_{J}$ is the face of $s_{I}$ and $s_{I^{\prime}}$. Moreover Masuda showed ([M], Lemma 4.4) that $s_{I}$ and $s_{I^{\prime}}$ lie on different sides of $s_{I} \cap s_{I^{\prime}}$. This implies that $\pi \mid s_{I} \cup s_{I^{\prime}}$ is injective and hence the orientability of $\left|\Sigma_{M}\right|$.

Lemma 2.4. The degree of $\pi:\left|\Sigma_{M}\right| \rightarrow H_{2}(B \bar{T} ; \mathbf{R}) \backslash\{0\}$ is equal to $T[M]$, the Todd genus of $M$.

This is essentially a restatement of $[\mathrm{M}]$, Theorem 4.2 paraphrased by using the projection $\pi$.

Lemma 2.5 ([M], Lemma 3.2). The equivariant first Chern class $c_{1}^{\bar{T}}(L)$ of a complex $\bar{T}$-line bundle $L$ over $M$ can be written in the form

$$
c_{1}^{\bar{T}}(L)=\sum c_{i} \xi_{i} \in \hat{H}_{\bar{T}}^{2}(M ; \mathbf{Z}) .
$$

Note. [M], Lemma 3.2 shows also that every element in $\hat{H}_{\bar{T}}^{2}(M ; \mathbf{Z})$ is of the form $c_{1}^{\bar{T}}(L)$. Similarly every element of $\hat{H}_{\bar{T}}^{2}(M ; \mathbf{R})$ can be written in the form $\sum c_{i} \xi_{i}$ with $c_{i} \in \mathbf{R}$.

For a complex $\bar{T}$-line bundle $L$ with $c_{1}^{\bar{T}}(L)=\sum c_{i} \xi_{i} \in \hat{H}_{\bar{T}}^{2}(M ; \mathbf{Z})$, we define the affine hyperplane $F_{i}$ in $H^{2}(B \bar{T} ; \mathbf{R})$ by

$$
F_{i}=\left\{u \in H^{2}(B \bar{T} ; \mathbf{R}) ;<u, v_{i}>=c_{i}\right\} .
$$

We set $F_{I}=\bigcap_{i \in I} F_{i}$ for $I \in \Sigma_{M}^{k-1} . F_{I}$ is a point for $I \in \Sigma_{M}^{n-1}$.

The moment map $\Phi_{L}$ of $L$ is a $\bar{T}$-invariant map $\Phi_{L}: M \rightarrow H^{2}(B \bar{T} ; \mathbf{R})$ uniquely determined by the complex $\bar{T}$-line bundle $L$. It has the following properties.

LEMma 2.6 ([M], Lemma 6.5). $\Phi_{L}\left(M_{I}\right) \subset F_{I}$ for any $I \in \Sigma_{M}^{k-1}$.

We shall add the following assumption:

All isotropy subgroups of the $T$ action are subtori, and each fixed point set component of subtori contains a point in $M^{T}$.

With this assumption the quotient space $M / T$ becomes a compact, connected orientable manifold of dimension $n$ with boundary. The boundary $\partial(M / T)$ is $\bigcup M_{i} / T$. Since the moment map $\Phi_{L}$ is $T$-invariant it factors through the map $\bar{\Phi}_{L}: M / T \rightarrow H^{2}(B \bar{T} ; \mathbf{R})$. Using this map $\bar{\Phi}_{L}$, the degree function

$$
d_{L}: H^{2}(B \bar{T} ; \mathbf{R}) \backslash \bigcup F_{i} \rightarrow \mathbf{Z}
$$

is defined as follows. Choose an orientation $o(\bar{T})$ of the torus $\bar{T}$, and define the orientation $o(M / T)$ of $M / T$ by requiring that $o(\bar{T})$ followed by $o(M / T)$ should coincide with $(-1)^{n(n-1) / 2}$ times that of $M$ as an almost complex manifold. The orientation $o(\bar{T})$ also determines that of $H^{2}(B \bar{T} ; \mathbf{R})$. Take $u \in H^{2}(B \bar{T} ; \mathbf{R}) \backslash \bigcup F_{i}$. Then $d_{L}(u)$ is the degree of

$$
H_{n}(M / T, \partial(M / T) ; \mathbf{Z}) \rightarrow H_{n}\left(H^{2}(B \bar{T} ; \mathbf{R}), H^{2}(B \bar{T} ; \mathbf{R}) \backslash\{u\} ; \mathbf{Z}\right) .
$$

This definition does not depend on the choice of $o(\bar{T})$. 
The function $d_{L}$ is locally constant. There is a transition formula for the values of $d_{L}$ when one moves from a component to another. Components of $H^{2}(B \bar{T} ; \mathbf{R}) \backslash \bigcup F_{i}$ will be called chambers. Two chambers $W_{\alpha}$ and $W_{\beta}$ are called adjacent if $\bar{W}_{\alpha} \cap \bar{W}_{\beta}$ has dimension $n-1$. In this case, let $F_{i}$ be such that $\bar{W}_{\alpha} \cap \bar{W}_{\beta} \subset F_{i}$. Then $\bar{W}_{\alpha} \cap \bar{W}_{\beta}$ is the closure of a component $W_{\alpha \beta}$ of $F_{i} \backslash \bigcup_{F_{i} \neq F_{j}} F_{j} . W_{\alpha \beta}$ will be called a wall between $W_{\alpha}$ and $W_{\beta}$. Note that there may be $F_{j}$ with $j \neq i, j \in \Sigma_{M}^{0}$ but $F_{j}=F_{i}$.

The transition formula is stated in the following way.

Lemma 2.7 ([KT], Remark 6.5; [M], Lemma 6.9). Let $W_{\alpha}$ and $W_{\beta}$ be adjacent chambers. Take points $u_{\alpha} \in W_{\alpha}$ and $u_{\beta} \in W_{\beta}$ such that the segment $\overline{u_{\alpha} u_{\beta}}$ from $u_{\alpha}$ to $u_{\beta}$ crosses the wall $W_{\alpha \beta}$ transversally. Then

$$
d_{L}\left(u_{\alpha}\right)=d_{L}\left(u_{\beta}\right)+\sum_{F_{i} \supset W_{\alpha \beta}} \operatorname{sign}<u_{\beta}-u_{\alpha}, v_{i}>d_{L \mid M_{i}}\left(u_{\alpha \beta i}\right),
$$

where $u_{\alpha \beta i}=\overline{u_{\alpha} u_{\beta}} \cap F_{i}$.

Here $d_{L \mid M_{i}}$ has to be understood as follows. Let $\bar{S}_{i}$ be the subcircle which stabilizes points in $M_{i}$ as before. Set $\bar{T}_{i}=\bar{T} / \bar{S}_{i}$. Then $\bar{T}_{i}$ acts effectively on $M_{i}$. Take a point $p \in M_{i} \cap M^{T}$ and put $\gamma=c_{i} \xi_{i} \mid p \in \operatorname{Hom}\left(\bar{T}, S^{1}\right)$. The restriction of $\gamma$ to $\operatorname{Hom}\left(\bar{S}_{i}, S^{1}\right)$ does not depend on the choice of $p$ in $M_{i} \cap M^{T}$, and $\bar{S}_{i}$ acts trivially on $L \chi^{-\gamma} \mid M_{i}$. Hence $L \chi^{-\gamma} \mid M_{i}$ can be regarded as a $\bar{T}_{i^{-}}$line bundle, and $d_{L \chi^{-\gamma \mid M_{i}}}$ is defined. If $u$ lies in $F_{i} \backslash \bigcup_{j \neq i} F_{j}$ we define

$$
d_{L \mid M_{i}}(u)=d_{L \chi^{-\gamma \mid M_{i}}}(u-\gamma)
$$

Note that $u-\gamma$ is in $H^{2}\left(B \bar{T}_{i} ; \mathbf{R}\right)$. It is easy to show that $d_{L \mid M_{i}}(u)$ is well-defined independently of the choice of $p$.

Lemma 2.8 ([M], Theorem 3.1). Let $K=\bigwedge^{n} T^{*} M$ be the canonical line bundle of $M$. The equivariant Chern class of $K$ considered as an element of $\hat{H}_{\bar{T}}^{2}(M ; \mathbf{Z})$ is given by

$$
c_{1}^{\bar{T}}(L)=-\sum \xi_{i} \in \hat{H}_{\bar{T}}^{2}(M ; \mathbf{Z}) .
$$

Define $\Phi_{L}^{\prime}: M \rightarrow H^{2}(B \bar{T} ; \mathbf{R})$ by

$$
\Phi_{L}^{\prime}=\Phi_{L}-\frac{1}{2} \Phi_{K}
$$

For each $i \in \Sigma_{M}^{0}$ the affine hyperplane $F_{i}^{\prime}$ is defined by

$$
F_{i}^{\prime}=\left\{u \in H^{2}(B \bar{T} ; \mathbf{R}) ;<u, v_{i}>=c_{i}+1 / 2\right\} .
$$

From 2.6 and 2.8 it follows that $\Phi_{L}^{\prime}\left(M_{i}\right)$ is contained in $F_{i}^{\prime}$. The degree function

$$
d_{L}^{\prime}: H^{2}(B \bar{T} ; \mathbf{R}) \backslash \bigcup F_{i}^{\prime} \rightarrow \mathbf{Z}
$$

is defined by using $\Phi_{L}^{\prime}$ in a similar way as $d_{L}$. Note that $H^{2}(B \bar{T} ; \mathbf{Z})$ is contained in $H^{2}(B \bar{T} ; \mathbf{R}) \backslash \bigcup F_{i}^{\prime}$

We are now in a position to state the main result of $[\mathrm{M}]$. Once a $\bar{T}$-invariant metric on $\mathrm{M}$ and a $\bar{T}$-invariant $U(1)$-connection of $L$ are given, the Dirac operator $D_{L}$ of the almost complex manifold $M$ twisted by the line bundle $L$ is defined. Its index, ind $D_{L}$, is a $\bar{T}$-module. In the topological context it is expressed as the image $\pi_{*}(L)$ of $L$ by the 
Gysin homomorphism $\pi_{*}: K_{\bar{T}}(M) \rightarrow K_{\bar{T}}(p t)=R(\bar{T})$, where $R(\bar{T})$ is the character ring of $\bar{T}$. It is identified with the group ring of $\operatorname{Hom}\left(\bar{T}, S^{1}\right)$ over $\mathbf{Z}$.

Theorem $2.9\left([\mathrm{M}]\right.$, Theorem 7.2; $[\mathrm{KT}]$, Theorem 2). If we write ind $D_{L}$ as

then $m(u)=d_{L}^{\prime}(u)$.

$$
\text { ind } D_{L}=\sum_{u \in H^{2}(B \bar{T} ; \mathbf{Z})} m(u) \chi^{u} \text {, }
$$

This finishes the review of $[\mathrm{M}]$.

3. Map $\Psi$. As we saw in the previous section the degree function $d_{L}$ was defined by using the moment map of $L$. It is desirable to explain it by using only combinatorial data of the simplicial set $\Sigma_{M}$ and the numbers $c_{i}$ associated with $i \in \Sigma_{M}^{0}$ which describe the $T$-line bundle $L$. The aim of this section is to give such an explanation. This is a joint work with Masuda. The results can be extended to cover unitary toric manifolds. So we will only give statement of results and sketch of proof here leaving the details elsewhere.

Let $\Sigma_{M}^{\prime}$ be the first barycentric subdivision of $\Sigma_{M}$ and $S_{M}=\left|\Sigma_{M}^{\prime}\right|$ the realization of $\Sigma_{M}^{\prime}$. The barycenter of $I \in \Sigma_{M}^{k-1}$ is denoted by $b_{I}$. These barycenters form the set of vertices of $\Sigma_{M}^{\prime}$. A simplex of $\Sigma_{M}^{\prime}$ is of the form

$$
\left(b_{I_{1}}, b_{I_{2}}, \ldots, b_{I_{l}}\right) \text { with } I_{1} \subset I_{2} \subset \ldots \subset I_{l} .
$$

The realization of $\left(b_{I_{1}}, \ldots, b_{I_{l}}\right)$ in $S_{M}$ will be denoted by $\left|b_{I_{1}}, \ldots, b_{I_{l}}\right|$. For each $I \in \Sigma_{M}^{k-1}$ we set

$$
\sigma_{I}=\bigcup_{I_{1}=I}\left|b_{I_{1}}, \ldots, b_{I_{l}}\right| \subset S_{M}
$$

It is called the dual cell of $I \in \Sigma_{M}^{k-1}$. When $I=\{i\} \in \Sigma_{M}^{0}$ we simply write $\sigma_{i}$ for $\sigma_{I}$. We see that $\sigma_{I} \subset \sigma_{J}$ if $I \supset J$. Also, if $I \cap I^{\prime}=\emptyset$ and $I \cup I^{\prime}$ is a simplex of $\Sigma_{M}$, then $\sigma_{I} \cap \sigma_{I^{\prime}}=\sigma_{I \cup I^{\prime}}$. The set of all dual cells $\left\{\sigma_{I}\right\}$ stratifies the complex $S_{M}$. In particular

$$
S_{M}=\bigcup_{i \in \Sigma_{M}^{0}} \sigma_{i} .
$$

Let $L k_{\Sigma_{M}} I$ be the link of $I$ in $S_{M}$ for $I \in \Sigma_{M}^{k-1}$. It is a simplicial set whose vertices are those $j \in \Sigma_{M}^{0}$ such that $j \notin I$ and $\{j\} \cup I \in \Sigma_{M}^{k}$, and whose simplices are those $J \in \Sigma_{M}^{l-1}$ such that $I \cap J=\emptyset$ and $I \cup J \in \Sigma_{M}^{k+l-1}$.

LEMmA 3.1. The boundary $\partial \sigma_{I}$ of the dual cell $\sigma_{I}$ is the realization of a simplicial set isomorphic to the first barycentric subdivision $L k_{\Sigma_{M}}^{\prime} I$ of $L k_{\Sigma_{M}} I$.

Proof. The boundary is the realization of a simplicial set $\Sigma(I)$ whose simplices are those $\left(b_{I_{1}}, \ldots, b_{I_{l}}\right)$ with $I \subset I_{1}$ but $I \neq I_{1}$ and $I_{1} \subset \cdots \subset I_{l}$. The correspondence which sends each simplex $\left(b_{J_{1}}, \ldots, b_{J_{l}}\right)$ of $L k_{\Sigma_{M}}^{\prime} I$ into $\left(b_{I \cup J_{1}}, \ldots, b_{I \cup J_{l}}\right)$ is an isomorphism of simplicial sets between $L k_{\Sigma_{M}}^{\prime} I$ and $\Sigma(I)$.

Lemma 3.2. The simplicial set $L k_{\Sigma_{M}} I$ is isomorphic to $\Sigma_{M_{I}}$ for any $I \in \Sigma_{M}^{k-1}$.

Proof. Take $i \in L k_{M}^{0} I$. Then $M_{I, i}=M_{I} \cap M_{i}$ is a characteristic submanifold of $M_{I}$ by virtue of the assumption (2.1). The vertex map from $L k_{M}^{0} I$ to $\Sigma_{M_{I}}^{0}$ which sends $i$ into $(I, i)$ gives the desired isomorphism. 
As an immediate corollary of Lemmas 3.1 and 3.2 we obtain

Corollary 3.3. $\partial \sigma_{I}$ is homeomorphic to $S_{M_{I}}$.

Given a collection $\hat{c}=\left\{c_{i}\right\}$ indexed by $\Sigma_{M}^{0}$ we define a map

$$
\Psi_{\hat{c}}: S_{M} \rightarrow H^{2}(B \bar{T} ; \mathbf{R})
$$

in the following way. It will be affine on each simplex $\left|b_{I_{1}}, \ldots, b_{I_{l}}\right|$ of $S_{M}$. Hence it is sufficient to assign a value for each vertex $b_{I}$. We do this by descending induction on the dimension $k-1$ of $I \in \Sigma_{M}^{k-1}$. When $k=n, \Psi_{\hat{c}}\left(b_{I}\right)$ is determined by the equation

$$
<\Psi_{\hat{c}}\left(b_{I}\right), v_{i}>=c_{i} \text { for } i \in I \text {. }
$$

Note that $\left\{v_{i} ; i \in I\right\}$ is a basis of $H_{2}(B \bar{T} ; \mathbf{Z})$ by Lemma 2.1. For $I \in \Sigma_{M}^{k-1}$ with $0<k<n$ we set $C_{I}=\left\{J \in \Sigma_{M}^{k} ; I \subset J\right\}$ and define

$$
\Psi_{\hat{c}}\left(b_{I}\right)=\frac{1}{\# C_{I}} \sum_{J \in C_{I}} \Psi_{\hat{c}}\left(b_{J}\right),
$$

where $\# C_{I}$ is the cardinality of $C_{I}$. The affine hyperplanes $F_{i}$ and $F_{I}$ are defined in a similar manner as in Section 2. As a direct consequence of the definition we have:

LEMMA 3.4. $\Psi_{\hat{c}}\left(\sigma_{I}\right) \subset F_{I}$ for any $I \in \Sigma_{M}^{k-1}$. In particular $\Psi_{\hat{c}}\left(S_{M}\right) \subset \bigcup F_{i}$.

Let $u$ be a point in $H^{2}(B \bar{T} ; \mathbf{R}) \backslash \bigcup F_{i}$. We define $\bar{d}_{\hat{c}}(u) \in \mathbf{Z}$ as the degree of the homomorphism

$$
\Psi_{\hat{c} *}: H_{n-1}\left(S_{M} ; \mathbf{Z}\right) \rightarrow H_{n-1}\left(H^{2}(B \bar{T} ; \mathbf{R}) \backslash\{u\} ; \mathbf{Z}\right) .
$$

Note that a preferred orientation of $H_{2}(B \bar{T} ; \mathbf{R})$ determines those of $S_{M}$ and $H^{2}(B \bar{T} ; \mathbf{R})$ simultaneously. Thus $\bar{d}_{\hat{c}}(u)$ is defined independently of the choice of orientations of $H_{2}(B \bar{T} ; \mathbf{R})$. When $\hat{c}$ comes from a $T$-line bundle $L$, i.e. when

$$
c_{1}^{\bar{T}}(L)=\sum c_{i} \xi_{i} \in \hat{H}_{\bar{T}}^{2}(M ; \mathbf{Z})
$$

we write $\bar{d}_{L}$ for $\bar{d}_{\hat{c}}$.

Theorem 3.5. The function $\bar{d}_{L}: H^{2}(B \bar{T} ; \mathbf{R}) \backslash \bigcup F_{i} \rightarrow \mathbf{Z}$ coincides with $d_{L}$.

Before proceeding to the proof we shall make some comments concerning the function $\bar{d}_{\hat{c}}$. At this point and hereafter we shall identify $\hat{c}=\left\{c_{i}\right\}$ with the cohomology class $\hat{c}=\sum_{i} c_{i} \xi_{i} \in \hat{H}_{\bar{T}}^{2}(M ; \mathbf{R})$.

Given an element $\gamma \in H^{2}(B \bar{T} ; \mathbf{R})$ we put

$$
\hat{c}^{\gamma}=\left\{c_{i}^{\prime}\right\} \text { with } c_{i}^{\prime}=c_{i}-<\gamma, v_{i}>
$$

and

$$
F_{i}^{\gamma}=\left\{u ;<u, v_{i}>=c_{i}^{\prime}\right\}
$$

for each $i$. The translation by $-\gamma$ sends the hyperplane $F_{i}$ to $F_{i}^{\gamma}$.

Assertion 3.6. If we regard $\hat{c}=\sum c_{i} \xi_{i}$ and $\hat{c}^{\gamma}=\sum c_{i}^{\prime} \xi_{i}$ as elements in $\hat{H}_{\bar{T}}^{2}(M ; \mathbf{R})$, then $\hat{c}^{\gamma}=\hat{c}-\gamma$. If $u$ is in $H^{2}(B \bar{T} ; \mathbf{R}) \backslash \bigcup F_{i}$, then $u-\gamma$ is in $H^{2}(B \bar{T} ; \mathbf{R}) \backslash \bigcup F_{i}^{\gamma}$, and

$$
\bar{d}_{\hat{c}}(u)=\bar{d}_{\hat{c} \gamma}(u-\gamma) \text {. }
$$


Proof. By Lemma 2.2, $\gamma=\sum<\gamma, v_{i}>\xi_{i}$ as elements in $\hat{H}_{\bar{T}}^{2}(M ; \mathbf{R})$. Hence

$$
\hat{c}-\gamma=\sum\left(c_{i}-<\gamma, v_{i}>\right) \xi_{i}=\hat{c}^{\gamma} .
$$

Then it is easy to see that that $\Psi_{\hat{c} \gamma}$ is the composition of $\Psi_{\hat{c}}$ and the translation by $-\gamma$. The identity above for the degree function $\bar{d}_{\hat{c}}$ follows readily from this observation.

Take $i \in \Sigma_{M}^{0}$ and take a vector $\gamma$ in $F_{i}$. Then $F_{i}^{\gamma}$ is identified with $H^{2}\left(B \bar{T}_{i} ; \mathbf{R}\right) \subset$ $H^{2}(B \bar{T} ; \mathbf{R})$. We set

$$
\hat{c}^{\gamma_{i}} \mid M_{i}=\left\{c_{j}^{\prime} ; j \in \Sigma_{M_{i}}\right\},
$$

and define $\Psi_{\hat{c}^{\gamma_{i} \mid M_{i}}}: S_{M_{i}} \rightarrow H^{2}\left(B \bar{T}_{i} ; \mathbf{R}\right)$ as before. From this the degree function

$$
\bar{d}_{\hat{c}^{\gamma_{i} \mid M_{i}}}: H^{2}\left(B \bar{T}_{i} ; \mathbf{R}\right) \backslash \bigcup_{j \in \Sigma_{M_{i}}} H^{2}\left(B \bar{T}_{i} ; \mathbf{R}\right) \cap F_{j}^{\gamma} \rightarrow \mathbf{Z}
$$

is induced as before.

Assertion 3.7. If we regard $\hat{c}$ as an element of $\hat{H}_{\frac{2}{T}}^{2}(M ; \mathbf{R})$ then $\hat{c}^{\gamma_{i}} \mid M_{i}$ is nothing but the restriction of $\hat{c}^{\gamma}$ to $M_{i}$. In particular, in case $\hat{c}$ comes from a $T$-line bundle $L$ and $\gamma$ lies in $H^{2}(B \bar{T} ; \mathbf{Z}) \cap F_{i}, \hat{c}^{\gamma_{i}} \mid M_{i}$ coincides with $\bar{d}_{L \chi^{-\gamma \mid M_{i}}}$.

Proof. We see that $\xi_{j} \mid M_{i}=0$ if $M_{i} \cap M_{j}=\emptyset$ by definition of $\xi_{j}$. Hence

$$
\hat{c}\left|M_{i}=\sum_{j \in L k_{\Sigma_{M}}^{0}\{i\}} c_{j} \xi_{j}\right| M_{i}+c_{i} \xi_{i} \mid M_{i} .
$$

Moreover $\xi_{j} \mid M_{i}$ belongs to $\hat{H}_{\bar{T}_{i}}^{2}\left(M_{i} ; \mathbf{Z}\right) \subset \hat{H}_{\bar{T}}^{2}\left(M_{i} ; \mathbf{Z}\right)$ because $M_{i} \cap M_{j}$ is a characteristic submanifold of $M_{i}$ for $j \in L k_{\Sigma_{M}}^{0}\{i\}$ under the assumption (2.1).

On the other hand, by Lemma 2.2

$$
\gamma=\sum_{j \in L k_{\Sigma_{M}}^{0}\{i\}}<\gamma, v_{j}>\xi_{j}\left|M_{i}+<\gamma, v_{i}>\xi_{i}\right| M_{i} .
$$

Since $<\gamma, v_{i}>=c_{i}$ we obtain

$$
\hat{c}^{\gamma_{i}}\left|M_{i}=\hat{c}\right| M_{i}-\gamma=\sum_{j \in L k_{\Sigma_{M}}^{0}\{i\}}\left(c_{j}-<\gamma, v_{j}>\right) \xi_{j}\left|M_{i}=\sum_{j \in L k_{\Sigma_{M}}^{0}\{i\}} c_{j}^{\prime} \xi_{j}\right| M_{i}
$$

Here is an analogue of Lemma 2.7:

LEMMA 3.8. Let $W_{\alpha}$ and $W_{\beta}$ be adjacent chambers and $u_{\alpha}$ and $u_{\beta}$ be such points that the segment $\overline{u_{\alpha} u_{\beta}}$ crosses the wall $W_{\alpha \beta}$ transversally. Then

$$
\bar{d}_{\hat{c}}\left(u_{\alpha}\right)=\bar{d}_{\hat{c}}\left(u_{\beta}\right)+\sum_{F_{i} \supset W_{\alpha \beta}} \operatorname{sign}<u_{\beta}-u_{\alpha}, v_{i}>\bar{d}_{\hat{c}^{\gamma_{i} \mid M_{i}}}\left(u_{\alpha \beta i}-\gamma_{i}\right)
$$

where $\gamma_{i}$ is any vector in $F_{i}$.

If Lemma 3.8 is admitted for a moment, then Theorem 3.5 can be deduced as follows. Lemma 2.7, Lemma 3.8 and Assertion 3.7 show that $d_{L}$ and $\bar{d}_{L}$ satisfy the same transition formula. Moreover, if $u \in H^{2}(B \bar{T} ; \mathbf{R})$ sits far away, then $d_{L}(u)=0=\bar{d}_{L}(u)$. Therefore $d_{L}$ and $\bar{d}_{L}$ coincide everywhere.

Lemma 3.8 itself follows from 
Lemma 3.9. Let $W$ be a chamber and $u \in W$. Let $r$ be a generic ray starting from $u$ with direction vector $\beta$, i.e. $r=\{u+\beta t ; t \geq 0\}$. Then

$$
\bar{d}_{\hat{c}}(u)=\sum_{i: F_{i} \cap r \neq \emptyset} \operatorname{sign}<\beta, v_{i}>\bar{d}_{\hat{c}^{\gamma_{i}} \mid M_{i}}\left(F_{i} \cap r-\gamma_{i}\right) .
$$

We here state the most crucial fact for proving Lemma 3.9. We identify $\Sigma_{M_{i}}$ with $L k_{\Sigma_{M}}\{i\}$ by Lemma 3.2, and then identify $S_{M_{i}}$ with $\partial \sigma_{i}$ by Lemma 3.1. This identification extends to an identification of the cone $C S_{M_{i}}$ over $S_{M_{i}}$ with $\sigma_{i}$, the vertex $o$ of the cone being identified with $b_{i} \in \sigma_{i}$. Let $\gamma_{i} \in F_{i}$. Then $\Psi_{\hat{c}^{\gamma_{i} \mid M_{i}}}$ is extended to a map $\tilde{\Psi}_{\hat{c}^{\gamma_{i} \mid M_{i}}}: \sigma_{i} \rightarrow H^{2}\left(B \bar{T}_{i} ; \mathbf{R}\right)$ sending $o$ to $\Psi_{\hat{c}}\left(b_{i}\right)-\gamma_{i}$. With this understood, we have

ASSERTION 3.10. $\tilde{\Psi}_{\hat{c}^{\gamma} \mid M_{i}}(x)+\gamma_{i}=\Psi_{\hat{c}}(x)$ for $x \in \sigma_{i}$.

The rest of details are rather routine and will be given elsewhere.

Finally we shall indicate the relation between the degree function of $[\mathrm{KT}]$ and $\bar{d}_{\hat{c}}$. Note that the Lie algebra $L(\bar{T})$ of $\bar{T}$ is canonically identified with $H_{2}(B \bar{T} ; \mathbf{R})$ and the dual $L(\bar{T})^{*}$ with $H^{2}(B \bar{T} ; \mathbf{R})$. Let $\omega$ be a $\bar{T}$-invariant closed 2 -form over $M$ which admits a moment map, i.e. a map $\Phi_{\omega}: M \rightarrow L(\bar{T})^{*}=H^{2}(B \bar{T} ; \mathbf{R})$ satisfying

$$
d<\Phi_{\omega}, v>=-i(\underline{v}) \omega
$$

where $\underline{v}$ is the vector field (infinitesimal action) associated with $v \in L(\bar{T})$. The cohomology class $[\omega] \in H^{2}(M ; \mathbf{R})$ comes down from some $[\hat{\omega}] \in H_{\bar{T}}^{2}(M ; \mathbf{R})$ as was proved in $[\mathrm{AB}]$. The class $[\hat{\omega}]$ is uniquely determined modulo the image of $H_{\bar{T}}^{2}(p t ; \mathbf{R})=H^{2}(B \bar{T} ; \mathbf{R})$. The image of $[\hat{\omega}]$ in $\hat{H}_{\bar{T}}^{2}(M ; \mathbf{R})$ can be written in the form

$$
\hat{c}_{\omega}=\sum c_{i} \xi_{i}, c_{i} \in \mathbf{R}
$$

Karshon and Tolman defined a degree function

$$
d_{\omega}: H^{2}(B \bar{T} ; \mathbf{R}) \backslash \bigcup F_{i} \rightarrow \mathbf{Z}
$$

using the moment map $\Phi_{\omega}$, and they showed similar formulas as Lemma 3.8 and Lemma 3.9. Then a similar argument as in the proof of Theorem 3.5 yields

THEOREM 3.11. $d_{\omega}=\bar{d}_{\hat{c}_{\omega}}$.

4. Positive line bundles. We keep the assumption on almost complex toric manifolds made in Section 2. We are interested in giving a combinatorial interpretation of $c_{1}(L)^{n}[M]$ for a $T$-line bundle over $M$. Note that $c_{1}(L)$ comes from the equivariant class $c_{1}^{T}(L) \in H_{T}^{2}(M ; \mathbf{Z})$.

We begin with an observation concerning the evaluation on the fundamental class. Consider the digram

$$
H^{*}(M) \stackrel{p}{\longleftarrow} H_{T}^{*}(M) \stackrel{q}{\longrightarrow} \hat{H}_{T}^{*}(M),
$$

where $\hat{H}_{T}^{*}$ denotes the quotient by $S$-torsion classes as in Section 2 and Section 3 , the coefficient group is the integers $\mathbf{Z}$ or the reals $\mathbf{R}$, and $p$ and $q$ are obvious maps.

Lemma 4.1. Let $M$ be an oriented closed $T$-manifold. For any $x_{1}, \ldots, x_{l} \in H_{T}^{*}(M)$, the evaluation $p\left(x_{1}\right) \cdots p\left(x_{l}\right)[M]$ depends only on $q\left(x_{1}\right), \ldots, q\left(x_{l}\right)$. 
P r o of. Consider the commutative diagram:

$$
\begin{aligned}
H_{T}^{*}(M) \stackrel{p}{\longrightarrow} & H^{*}(M) \\
\pi_{*} \downarrow & \\
H^{*}(B T)= & H_{T}^{*}(p t) \stackrel{p}{\longrightarrow} H^{*}(p t)
\end{aligned}
$$

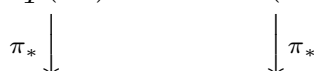

where $p t$ is one point and $\pi_{*}$ is the Gysin homomorphism. We have

$$
p\left(x_{1}\right) \cdots p\left(x_{l}\right)[M]=\pi_{*} p\left(x_{1} \cdots x_{l}\right)=p \pi_{*}\left(x_{1} \cdots x_{l}\right) .
$$

If $y_{1}, \ldots, y_{l}$ are $S$-torsion elements, then

$$
p\left(x_{1}+y_{1}\right) \cdots p\left(x_{l}+y_{l}\right)[M]=p \pi_{*}\left(x_{1} \cdots x_{l}\right)+p \pi_{*}(y),
$$

with $y$ an $S$-torsion element. Since $\pi_{*}$ is an $H_{T}^{*}(p t)$-module map, $\pi_{*}(y) \in H_{T}^{*}(p t)$ is also an $S$-torsion element, and hence $\pi_{*}(y)=0$.

Hereafter we will not distinguish $H_{T}^{*}(M)$ and $\hat{H}_{T}^{*}(M)$ as far as the evaluation on the fundamental class is concerned.

In Section 3 we considered $\bar{T}$-line bundles. In this section we consider more generally $T$-line bundles. If $L$ is a $T$-line bundle it is easy to see that there exists an element $\delta \in H^{2}(B T ; \mathbf{Z})$ such that the bundle $L \chi^{-\delta}$ comes from a $\bar{T}$-line bundle. If

$$
c_{1}^{\bar{T}}\left(L \chi^{-\delta}\right)=\sum c_{i} \xi_{i} \in \hat{H}_{\bar{T}}^{2}(M ; \mathbf{Z}), c_{i} \in \mathbf{Z},
$$

then

$$
c_{1}^{T}(L)=\sum c_{i} \xi_{i}+\delta \in \hat{H}_{T}^{2}(M ; \mathbf{Z}) .
$$

Note that we regard $\hat{H}_{\bar{T}}^{*}(M)$ as embedded in $\hat{H}_{T}^{*}(M)$.

More generally we consider $\hat{c} \in \hat{H}_{T}^{2}(M ; \mathbf{R})$ of the form

$$
\hat{c}=\sum c_{i} \xi_{i}+\delta, c_{i} \in \mathbf{R}, \delta \in H^{2}(B T ; \mathbf{R}) .
$$

Set

and define

$$
\hat{c}^{\delta}=\sum c_{i} \xi_{i} \in \hat{H}_{\bar{T}}^{2}(M ; \mathbf{R})
$$

$$
F_{i}^{\delta}=\left\{u \in H^{2}(B \bar{T} ; \mathbf{R}) ;<u, v_{i}>=c_{i}\right\} .
$$

Chambers with respect to $F_{i}^{\delta}$ are denoted by $W_{\alpha}^{\delta}, W_{\beta}^{\delta}$ and so on, and walls by $W_{\alpha \beta}^{\delta}$ and so on. $F_{i}, W_{\alpha}, W_{\alpha}, W_{\alpha \beta}$ will denote the subsets of $H^{2}(B \bar{T} ; \mathbf{R})+\delta$ obtained from $F_{i}^{\delta}, W_{\alpha}^{\delta}, W_{\beta}^{\delta}, W_{\alpha \beta}^{\delta}$ by the translation by $\delta$. For $u_{\alpha} \in W_{\alpha}$ we define

$$
\bar{d}_{\hat{c}}\left(u_{\alpha}\right)=\bar{d}_{\hat{c}^{\delta}}(u-\delta) .
$$

Since $\bar{d}_{\hat{c}}$ is constant on $W_{\alpha}$ we put $\bar{d}_{\hat{c}}\left(W_{\alpha}\right)=\bar{d}_{\hat{c}}\left(u_{\alpha}\right)$. When $\hat{c}=c_{1}^{T}(L)$ we write $\bar{d}_{L}$ for $\bar{d}_{\hat{c}}$ and $\bar{d}_{L}\left(W_{\alpha}\right)$ for $\bar{d}_{\hat{c}}\left(W_{\alpha}\right)$.

The lattice $H^{2}(B \bar{T} ; \mathbf{Z})$ in $H^{2}(B \bar{T} ; \mathbf{R})$ defines a measure on $H^{2}(B \bar{T} ; \mathbf{R})$. If $e_{1}, \ldots, e_{n}$ is a basis of $H^{2}(B \bar{T} ; \mathbf{Z})$, then the parallelotope spanned by $0, e_{1}, \cdots, e_{n}$ has measure 1 . The translation by $\delta$ transfers the measure on $H^{2}(B \bar{T} ; \mathbf{R})$ over $H^{2}(B \bar{T} ; \mathbf{R})+\delta$. The volume of a subset $A$ of $H^{2}(B \bar{T} ; \mathbf{R})$ or $H^{2}(B \bar{T} ; \mathbf{R})+\delta$ is denoted by $|A|$. 
THEOREM 4.2. Let $\hat{c}$ be as above.Then

$$
p(\hat{c})^{n}[M]=n ! \sum \bar{d}_{\hat{c}}\left(W_{\alpha}\right)\left|W_{\alpha}\right|,
$$

where $[M]$ is the fundamental class of $M$, and the sum ranges over all chambers $W_{\alpha}$. Since $\bar{d}_{\hat{c}}\left(W_{\alpha}\right)=0$ for all unbounded chambers the above sum has a meaning.

Corollary 4.3. Let $L$ be a T-line bundle over $M$ with

$$
c_{1}^{T}(L)=\sum c_{i} \xi_{i}+\delta \in \hat{H}_{T}^{2}(M ; \mathbf{Z}), \delta \in H^{2}(B T ; \mathbf{Z}) .
$$

Then

$$
c_{1}(L)^{n}[M]=n ! \sum \bar{d}_{L}\left(W_{\alpha}\right)\left|W_{\alpha}\right|,
$$

REMARK 4.4. Theorem 4.2 can be regarded as a combinatorial counterpart of Theorem 1 in $[\mathrm{KT}]$. Karshon and Tolman expressed the push-forward $\left(\Phi_{\omega}\right)_{*} \omega^{n}$ of the Liouville measure by a formula corresponding to Theorem 4.2 .

For the proof of Theorem 4.2 we use a purely combinatorial lemma. To state the lemma we need some preliminaries. Let $V$ be a real vector space with a lattice $N$ in $V$. Let $V^{*}$ be the dual space of $V$ and $N^{*}$ the dual lattice of $N$ in $V^{*}$. The lattice $N^{*}$ defines a measure on $V^{*}$ as above. Let $v$ be a primitive vector in $N$. Then $N_{v}^{*}=\left\{u \in N^{*} ;\langle u, v\rangle=0\right\}$ is a lattice in $V_{v}^{*}=\left\{u \in V^{*} ;\langle u, v\rangle=0\right\}$, and hence determines a measure in $V_{v}^{*}$. If $c$ is a real number, then we transfer the measure on $V_{v}^{*}$ to the affine hyperplane $F_{v}=\left\{u \in V^{*} ;\langle u, v\rangle=c\right\}$ by translation by a vector lying in $F_{v}$. The volume of a subset $B \subset F_{v}$ is also denoted by $|B|$.

Let $v_{1}, \ldots, v_{d}$ be primitive vectors in $N$ and $\hat{c}=\left(c_{1}, \ldots, c_{d}\right)$ a sequence of real numbers. Define

$$
F_{i}=\left\{u ; u \in V^{*},\left\langle u, v_{i}>=c_{i}\right\} .\right.
$$

Chambers and walls with respect to these affine hyperplanes $F_{i}$ are defined as in Section 3 . Let $D$ be the union of the closures of certain bounded chambers. The boundary $\partial D$ of $D$ is the union of the closures of certain walls:

$$
\partial D=\bigcup D_{i_{k}}, D_{i_{k}} \subset F_{i_{k}} .
$$

Let $u_{i_{k}}$ be a vector which points outward from $D$ and crosses $D_{i_{k}}$ transversally. We set

$$
\epsilon_{i_{k}}=\operatorname{sign}<u_{i_{k}}, v_{i_{k}}>\text {. }
$$

LEMMA 4.5. $n|D|=\sum \epsilon_{i_{k}} c_{i_{k}}\left|D_{i_{k}}\right|$.

Pro of. Let $x_{1}, \ldots, x_{n}$ be a coordinate system of $V^{*}$ with respect to a basis $e_{1}, \ldots, e_{n}$ of $N^{*}$. Define an $(n-1)$-form $\omega$ on $V^{*}$ by

$$
\omega=\sum(-1)^{j-1} x_{j} d x_{1} \wedge \cdots \wedge d \hat{x}_{j} \wedge \cdots \wedge d x_{n} .
$$

The form $\omega$ is $S L(n, \mathbf{R})$-invariant so that it does not depend on the choice of integral basis $e_{1}, \ldots, e_{n}$. By the Stokes theorem

$$
\int_{\partial D} \omega=n \int_{D} d x_{1} \cdots d x_{n}=n|D|
$$


where $V^{*}$ is oriented by $d x_{1} \wedge \cdots \wedge d x_{n}$. On the other hand

$$
\int_{\partial D} \omega=\sum_{i_{k}} \int_{D_{i_{k}}} \omega
$$

Once $i_{k}$ is fixed, we can take $e_{1}, \ldots, e_{n}$ so that $x_{1}=v_{i_{k}}$ on $D_{i_{k}}$. Then $\omega$ takes the form $\omega=x_{1} d x_{2} \wedge \ldots \wedge d x_{n}$ on $D_{i_{k}}$. Comparing the orientations of $F_{i}$ determined by $d x_{2} \wedge \cdots \wedge d x_{n}$ and of $D_{i_{k}} \subset \partial D$ we obtain

Hence $n|D|=\sum \epsilon_{i_{k}} c_{i_{k}}\left|D_{i_{k}}\right|$.

$$
\int_{D_{i_{k}}} \omega=\epsilon_{i_{k}} c_{i_{k}}\left|D_{i_{k}}\right|
$$

Proof of Theorem 4.2. Clearly we may assume that $\hat{c}$ lies in $\hat{H}_{\bar{T}}^{2}(M ; \mathbf{R})$, so that $\hat{c}=$ $\sum c_{i} \xi_{i}$. Proof will proceed by induction on $n$.

First consider the case $n=1$. In this case $M$ is isomorphic to the projective line with non-trivial torus action, and $M^{T}$ consists of two points $p_{1}, p_{2}$. Moreover $v_{1}, v_{2}$ satisfy $v_{1}+v_{2}=0$. Define $u_{1}, u_{2} \in H^{2}(B \bar{T} ; \mathbf{R})$ by

$$
<u_{1}, v_{1}>=c_{1},<u_{2}, v_{2}>=c_{2}
$$

i.e.

$$
<u_{1}, v_{1}>=c_{1},<u_{2}, v_{1}>=-c_{2} .
$$

Then $F_{1}=\left\{u_{1}\right\}$ and $F_{2}=\left\{u_{2}\right\}$. If $u_{1}=u_{2}$ i.e. $c_{1}+c_{2}=0$ then there is no bounded chamber. If $u_{1} \neq u_{2}$ then there is a unique bounded chamber $W$ and $\bar{d}_{\hat{c}}(W)=\operatorname{sign}\left(c_{1}+c_{2}\right)$ and $|W|=\left|c_{1}+c_{2}\right|$. Hence $\bar{d}_{\hat{c}}(W)|W|=c_{1}+c_{2}$.

On the other hand

$$
\hat{c}=c_{1} \xi_{1}+c_{2} \xi_{2}
$$

But, by Lemma 2.2,

$$
u_{2}=<u_{2}, v_{1}>\xi_{1}+<u_{2}, v_{2}>\xi_{2}=c_{2}\left(-\xi_{1}+\xi_{2}\right) .
$$

Hence $\hat{c}=\left(c_{1}+c_{2}\right) \xi_{1}+u_{2} \in H_{\bar{T}}^{2}(M ; \mathbf{R})$ and $p(\hat{c})=\left(c_{1}+c_{2}\right) p\left(\xi_{1}\right)$. Since $\xi_{1}$ is the Poincaré dual of a point in $M$, we have

$$
p\left(\xi_{1}\right)[M]=1 \text { and } p(\hat{c})[M]=c_{1}+c_{2} .
$$

Thus

$$
p(\hat{c})[M]=\bar{d}_{\hat{c}}(W)|W| .
$$

Now suppose $n>1$. For the sake of simplicity we write $\hat{c}^{n}[M]$ for $p(\hat{c})^{n}[M]$ and so on. We have

$$
\hat{c}^{n}[M]=\left(\hat{c}^{n-1} \sum_{i} c_{i} \xi_{i}\right)[M]=\sum_{i} c_{i} \hat{c}^{n-1}\left[M_{i}\right]
$$

since $\xi_{i}$ is the Poincaré dual of $M_{i}$ in $M$. By the inductive assumption we get

$$
\hat{c}^{n}[M]=(n-1) ! \sum_{i} c_{i} \sum_{\alpha} \bar{d}_{\hat{c} \mid M_{i}}\left(W_{i \alpha}\right)\left|W_{i \alpha}\right| .
$$

Here $\bar{d}_{\hat{c} \mid M_{i}}\left(W_{i \alpha}\right)$ has the following meaning. We define $\gamma_{i}$ and $\hat{c}^{\gamma_{i}} \mid M_{i}$ as in Section 3. Note that $p\left(\hat{c}^{\gamma_{i}} \mid M_{i}\right)=p\left(\hat{c} \mid M_{i}\right)$. The $\left\{W_{i \alpha}\right\}$ are chambers in $H^{2}\left(B \bar{T}_{i} ; \mathbf{R}\right)$ with respect to $\hat{c}^{\gamma_{i}} \mid M_{i}$. We write $\bar{d}_{\hat{c} \mid M_{i}}\left(W_{i \alpha}\right)$ for $\bar{d}_{\hat{c} \gamma_{i} \mid M_{i}}\left(W_{i \alpha}\right)$. As in Section 3 this has an invariant 
meaning independent of $\gamma_{i}$. If one translates $W_{i \alpha}$ by the vector $\gamma_{i}$, then the image is a connected component of $F_{i} \backslash \bigcup_{F_{j} \neq F_{i}} F_{j}$. We also denote this component by $W_{i \alpha}$.

As a subset of $F_{i}$ the closure $\bar{W}_{i \alpha}$ of $W_{i \alpha}$ is the union of the closures of certain walls. The closure of a wall $W_{\beta \beta^{\prime}}$ will be denoted by $D_{\beta \beta^{\prime}}$. When we particularly want to regard $D_{\beta \beta^{\prime}}$ as sitting in $F_{i}$ we use the notation $D_{\beta \beta^{\prime} i}$. To proceed further, the following observation is in order.

Suppose that $F_{i}$ and $F_{i^{\prime}}$ with $i \neq i^{\prime}$ both contain $D_{\beta \beta^{\prime}}$. It means in particular that $F_{i}=F_{i^{\prime}}$ as hyperplanes. Then, either $v_{i}=v_{i^{\prime}}$ and $c_{i}=c_{i^{\prime}}$ or $v_{i}=-v_{i^{\prime}}$ and $c_{i}=-c_{i^{\prime}}$, because $v_{i}$ and $v_{i^{\prime}}$ are primitive vectors in $H_{2}(B \bar{T} ; \mathbf{Z})$. Therefore the number

$$
c_{\beta \beta^{\prime}}=c_{i} \operatorname{sign}<u_{\beta^{\prime}}-u_{\beta}, v_{i}>, u_{\beta} \in W_{\beta}, u_{\beta^{\prime}} \in W_{\beta^{\prime}},
$$

depends only on $W_{\beta}$ and $W_{\beta^{\prime}}$. Note that

$$
c_{\beta \beta^{\prime}}=-c_{\beta^{\prime} \beta} .
$$

Now, in view of (4.1), Theorem 4.2 will follow from

Assertion 4.6.

$$
\sum_{i, \alpha} c_{i} \bar{d}_{\hat{c} \mid M_{i}}\left(W_{i \alpha}\right)\left|W_{i \alpha}\right|=n \sum_{\beta} \bar{d}_{\hat{c}}\left(W_{\beta}\right)\left|W_{\beta}\right| .
$$

Proof. When $D_{\beta \beta^{\prime} i}$ is contained in $W_{i \alpha}$, we set $\bar{d}_{\hat{c} \mid M_{i}}\left(D_{\beta \beta^{\prime} i}\right)=\bar{d}_{\hat{c} \mid M_{i}}\left(W_{i \alpha}\right)$. Then

$$
\sum_{i, \alpha} c_{i} \bar{d}_{\hat{c} \mid M_{i}}\left(W_{i \alpha}\right)\left|W_{i \alpha}\right|=\sum_{\beta, \beta^{\prime}, i} c_{i} \bar{d}_{\hat{c} \mid M_{i}}\left(D_{\beta \beta^{\prime} i}\right)\left|D_{\beta \beta^{\prime} i}\right|
$$

where the summation ranges over all $i \in \Sigma_{M}^{0}$ and all walls $W_{\beta \beta^{\prime}}$ lying in $F_{i}$. The right hand side of (4.2) can be put in the form

$$
=\sum_{\beta, \beta^{\prime}, i} \operatorname{sign}<u_{\beta \beta^{\prime}}, v_{i}>\bar{d}_{\hat{c} \mid M_{i}}\left(D_{\beta \beta^{\prime} i}\right) c_{\beta \beta^{\prime}}\left|D_{\beta \beta^{\prime}}\right|
$$

where $u_{\beta \beta^{\prime}}=u_{\beta^{\prime}}-u_{\beta}$. By the degree transition formula (Lemma 3.8)

$$
\sum_{i} \operatorname{sign}<u_{\beta \beta^{\prime}}, v_{i}>\bar{d}_{\hat{c} \mid M_{i}}\left(D_{\beta \beta^{\prime} i}\right)=\bar{d}_{\hat{c}}\left(W_{\beta}\right)-\bar{d}_{\hat{c}}\left(W_{\beta^{\prime}}\right) .
$$

Putting this in (4.3) and noting that $c_{\beta \beta^{\prime}}=-c_{\beta^{\prime} \beta},(4.3)$ is transformed into

$$
\sum_{\beta, \beta^{\prime}} \bar{d}_{\hat{c}}\left(W_{\beta}\right) c_{\beta \beta^{\prime}}\left|D_{\beta \beta^{\prime}}\right| \text {. }
$$

But, if one fixes $\beta$, then Lemma 4.5 tells us that

$$
\sum_{\beta^{\prime}} c_{\beta \beta^{\prime}}\left|D_{\beta \beta^{\prime}}\right|=n\left|W_{\beta}\right| .
$$

Hence the right hand side of (4.2) is finally transformed into

$$
n \sum_{\beta} \bar{d}_{\hat{c}}\left(W_{\beta}\right)\left|W_{\beta}\right| .
$$

This finishes the proof of Assertion 4.6 and hence Theorem 4.2.

Corollary 4.3 suggests the following definition of positiveness of $T$-line bundles over $M$. More generally, in view of Theorem 4.2, we define the positiveness of $\hat{c}=\sum c_{i} \xi_{i}+\delta \in$ 
$\hat{H}_{T}^{2}(M ; \mathbf{R}), \delta \in H^{2}(B T ; \mathbf{R})$, in the following way. In case $\hat{c}=c_{1}^{T}(L)$ and $\hat{c}$ is positive we call the $T$-line bundle $L$ positive. When $n=1$, using the notations in the proof of Theorem 4.2, we call $\hat{c}$ positive if $c_{1}+c_{2}>0$. This condition is equivalent to the condition $\hat{c}[M]>0$ by Theorem 4.2 .

Suppose $n>1$. We proceed by induction on the dimension $n$. We say that $\hat{c}$ is positive if the following conditions are satisfied:

1. $\hat{c} \mid M_{I}$ is positive for all $I \in \Sigma_{M}^{k-1}, 1 \leq k \leq n$.

2. $F_{i} \neq F_{j}$ for $i \neq j$.

3. One can go out from any chamber to an unbounded chamber by successively passing walls in positive direction.

Here we say that one can pass in positive direction from a chamber $W_{\alpha}$ to an adjacent chamber $W_{\beta}$ if $\left\langle u_{\beta}-u_{\alpha}, v_{i}>>0\right.$ for all $F_{i}$ containing the wall $W_{\alpha \beta}$, where $u_{\alpha} \in W_{\alpha}$ and $u_{\beta} \in W_{\beta}$.

Proposition 4.7. Suppose that $\hat{c}$ is positive. Then

1. $\bar{d}_{\hat{c}}\left(W_{\alpha}\right) \geq 0$ for all chambers $W_{\alpha}$.

2. $\hat{c}^{k}\left[M_{I}\right]>0$ for all $k, 1 \leq k \leq n$ and all $I \in \Sigma_{M}^{n-k-1}$.

When $k=n$, (2) means $\hat{c}^{n}[M]>0$.

In view of Theorem 3.11 and/or Theorem 3.5 we can restate Proposition 4.7 in terms of $d_{\hat{c}}$ and $/$ or $d_{L}$. For example

COROLlaRY 4.8. Let $L$ be a positive T-line bundle over $M$. Then

1. $d_{L}\left(W_{\alpha}\right) \geq 0$ for all chambers $W_{\alpha}$.

2. $c_{1}(L)^{k}\left[M_{I}\right]>0$ for all $k, 1 \leq k \leq n$ and all $I \in \Sigma_{M}^{n-k-1}$.

Proof of Proposition 4.7. Proof will proceed by induction. First we show that $\bar{d}_{\hat{c}}\left(W_{\alpha}\right) \geq$ 0 for all chambers $W_{\alpha}$. In the case $n=1$ we have $\bar{d}_{\hat{c}}\left(W_{\alpha}\right)=\operatorname{sign}\left(c_{1}+c_{2}\right)>0$ for the unique bounded chamber $W_{\alpha}$ and $\bar{d}_{\hat{c}}\left(W_{\beta}\right)=0$ for unbounded chambers $W_{\beta}$ as was shown in the proof of Theorem 4.2. In particular $\hat{c}[M]=c_{1}+c_{2}>0$ in this case.

Suppose $n>1$. The inductive assumption implies $\bar{d}_{\hat{c} \mid M_{i}}\left(W_{\beta \beta^{\prime}}\right) \geq 0$ for all $F_{i}$ and for all walls $W_{\beta \beta^{\prime}} \subset F_{i}$. Then, from the condition (3) of positiveness and the degree transition formula it follows that $\bar{d}_{\hat{c}}\left(W_{\alpha}\right) \geq 0$ for all chambers $W_{\alpha}$ since $\bar{d}_{\hat{c}}\left(W_{\beta}\right)=0$ for faraway unbounded chambers $W_{\beta}$. Theorem 4.2 then yields

$$
\hat{c}^{n}[M]>0 .
$$

For $I \in \Sigma_{M}^{n-k-1}$ with $1 \leq k<n, \hat{c} \mid M_{I}$ is positive by the condition (1) of positiveness. Thus, by inductive assumption, $\hat{c}^{k}\left[M_{I}\right]>0$.

REMark 4.9. As indicated at the end of Section 3 the equivariant cohomology class $\hat{c}$ can be thought of as representing a closed 2-form admitting moment map over $M$. In this context positive classes may be thought of as representing certain closed 2-forms participating in the positiveness property of symplectic forms.

REMARK 4.10. Corresponding to Remark 4.9 positive $T$-line bundles have property (2) of Corollary 4.8 analogous to ample line bundles over toric manifold. However the 
converse statement of Corollary 4.8 is not true in general. In section 5 , it will be shown, under the additional assumption $T[M]=1$, that the converse is also true. In this sense Corollary 4.8 may be regarded as an analogue of the Nakai criterion of ampleness of line bundles.

From the definition of positiveness we readily have the following

Proposition 4.11. If $\hat{c}$ is positive, then $r \hat{c}$ is also positive for any positive real number $r$. If $\left\{\epsilon_{i}\right\}$ is a collection of sufficiently small real numbers indexed by $\Sigma_{M}^{0}$ and $\hat{c}^{\prime}=\hat{c}+\sum_{i} \epsilon_{i} \xi_{i}$, then $\hat{c}^{\prime}$ is also positive.

We shall discuss some numerical necessary conditions for $\hat{c}$ to be positive. To begin with, assume $n \geq 2$ and take an $(n-2)$-simplex $J \in \Sigma_{M}^{n-2}$. Then $L k_{\Sigma_{M}} J$ consists of two points $i$ and $i^{\prime}$.

LEMMA 4.12. $v_{i}$ and $v_{i^{\prime}}$ are related by an equation of the following form:

$$
v_{i}+v_{i^{\prime}}=\sum_{j \in J} a_{j} v_{j}, a_{j} \in \mathbf{Z} .
$$

Proof. Let $J=\left\{j_{1}, \ldots, j_{n-1}\right\} . v_{i}, v_{j_{1}}, \ldots, v_{j_{n-1}}$ and $v_{i^{\prime}}, v_{j_{1}}, \ldots, v_{j_{n-1}}$ are both bases of $H_{2}(B \bar{T} ; \mathbf{Z})$. Therefore they are related by a linear relation

$$
\left(v_{i^{\prime}}, v_{j_{1}}, \ldots, v_{j_{n-1}}\right)=\left(v_{i}, v_{j_{1}}, \ldots, v_{j_{n-1}}\right)\left(\begin{array}{ccccc}
-1 & 0 & \cdots & & 0 \\
a_{j_{1}} & 1 & 0 & \cdots & 0 \\
& & \ddots & & \\
& & & \ddots & \\
& & & 0 & 1
\end{array}\right) .
$$

Here -1 comes because $v_{i}$ and $v_{i^{\prime}}$ sit on different sides of $s_{J}=\left|v_{j_{1}}, \ldots, v_{j_{n-1}}\right|$ by [M], Lemma 4.4, cf. also Lemma 2.3.

LEMMA 4.13. Under the same situation as in Lemma 4.12 we have:

$$
\begin{aligned}
& \xi_{i}\left[M_{J}\right]=\xi_{i^{\prime}}\left[M_{J}\right]=1, \\
& \xi_{j}\left[M_{J}\right]=-a_{j}, \quad j \in J, \\
& \xi_{k}\left[M_{J}\right]=0, \quad k \neq i, i^{\prime}, \quad k \notin J .
\end{aligned}
$$

Pro of (Due to Masuda). $p\left(\xi_{i} \mid M_{J}\right), p\left(\xi_{i^{\prime}} \mid M_{J}\right) \in H^{2}\left(M_{J} ; \mathbf{Z}\right)$ are the Poincaré dual in $M_{J}$ of a point as is easily seen. Therefore

$$
\xi_{i}\left[M_{J}\right]=\xi_{i^{\prime}}\left[M_{J}\right]=1 .
$$

If $k \neq i, i^{\prime}, k \notin J$, then $M_{k} \cap M_{J}=\emptyset$. Therefore $\xi_{k} \mid M_{J}=0$ and $\xi_{k}\left[M_{J}\right]=0$. But

We put $\xi_{J}=\prod_{j \in J} \xi_{j} \in \hat{H}_{\bar{T}}^{2(n-1)}(M ; \mathbf{Z})$. For any $u \in H^{2}(B \bar{T} ; \mathbf{R})$, we have $u \xi_{J}[M]=0$.

$$
u \xi_{J}[M]=\sum_{k}<u, v_{k}>\xi_{k} \xi_{J}[M]=\sum_{k}<u, v_{k}>\xi_{k}\left[M_{J}\right]
$$

by Lemma 2.2. Hence we get

$$
0=<u, v_{i}>+<u, v_{i^{\prime}}>+\sum_{j \in J}<u, v_{j}>\xi_{j}\left[M_{J}\right] .
$$


Then, by Lemma 4.12 ,

$$
0=\sum_{j \in J}<u, v_{j}>\left(a_{j}+\xi_{j}\left[M_{J}\right]\right) .
$$

Since this holds for any $u$, it follows that $\xi_{j}\left[M_{J}\right]=-a_{j}$ for all $j \in J$.

As an immediate consequence of Lemma 4.13 we obtain

Corollary 4.14. Suppose $n \geq 2$. Then

$$
\hat{c}\left[M_{J}\right]=c_{i}+c_{i^{\prime}}-\sum_{j \in J} c_{j} a_{j}
$$

for any $J \in \Sigma_{M}^{n-2}$.

As a direct consequence of Corollary 4.14 and Proposition 4.7 we obtain

Proposition 4.15. Suppose $n \geq 2$. Then $\hat{c}\left[M_{J}\right]>0$ if and only if

$$
c_{i}+c_{i^{\prime}}-\sum_{j \in J} c_{j} a_{j}>0
$$

for any $J \in \Sigma_{M}^{n-2}$. In particular if $\hat{c}$ is positive, then

$$
c_{i}+c_{i^{\prime}}-\sum_{j \in J} c_{j} a_{j}>0 \text { for all } J \in \Sigma_{M}^{n-2} .
$$

We keep the same situation as above and put

$$
I=\{i\} \cup J \text { and } I^{\prime}=\left\{i^{\prime}\right\} \cup J \in \Sigma_{M}^{n-1} .
$$

Put also $u_{I}=\Psi_{\hat{c}}\left(b_{I}\right)$ and $u_{I^{\prime}}=\Psi_{\hat{c}}\left(b_{I^{\prime}}\right)$. They are determined by

$$
\begin{aligned}
& <u_{I}, v_{i}>=c_{i}, \quad<u_{I}, v_{j}>=c_{j} \text { for } j \in J, \\
& <u_{I^{\prime}}, v_{i^{\prime}}>=c_{i^{\prime}}, \quad<u_{I^{\prime}}, v_{j}>=c_{j} \text { for } j \in J \text {. }
\end{aligned}
$$

LEMMA 4.16 .

$$
\begin{gathered}
<u_{I}-u_{I^{\prime}}, v_{i}>=<u_{I^{\prime}}-u_{I}, v_{i^{\prime}}>=c_{i}+c_{i^{\prime}}-\sum_{j \in J} a_{j} c_{j} . \\
<u_{I}-u_{I^{\prime}}, v_{j}>=0 \text { for } j \in J .
\end{gathered}
$$

Proof.

$<u_{I}-u_{I^{\prime}}, v_{i}>=c_{i}-<u_{I^{\prime}}, v_{i}>=c_{i}-<u_{I^{\prime}},-v_{i^{\prime}}+\sum a_{j} v_{j}>=c_{i}+c_{i^{\prime}}-\sum a_{j} c_{j}$.

Similar computations yield the other equalities.

Corollary 4.17. Suppose $\hat{c}\left[M_{J}\right]>0$. Then

$$
<u_{I}-u_{I^{\prime}}, v_{i}>=<u_{I^{\prime}}-u_{I}, v_{i^{\prime}}>>0 .
$$

In particular $<u_{I^{\prime}}, v_{i}><c_{i}$. Also $<\Psi_{\hat{c}}\left(b_{J}\right), v_{i}><c_{i}$.

Proof. For the third inequality note that $\Psi_{\hat{c}}\left(b_{J}\right)=\frac{1}{2}\left(u_{I}+u_{I^{\prime}}\right)$.

Recall that $s_{I}$ is the realization of $I \in \Sigma_{M}$ in $\left|\Sigma_{M}\right|$, and $\sigma_{i}$ is the dual cell in $S_{M}$ corresponding to $i \in \Sigma_{M}^{0}$. Two $(n-1)$-simplices $I, I^{\prime}$ will be called adjacent if $I \cap I^{\prime}$ belongs to $\Sigma_{M}^{n-2}$. 
Lemma 4.18. Suppose that $n \geq 2$ and fix $I \in \Sigma_{M}^{n-1}$. Let $I_{1}, \ldots, I_{l}$ be the totality of $(n-1)$-simplices such that $I$ and $I_{\nu}$ are adjacent. Put $J_{\nu}=I \cap I_{\nu} \in \Sigma_{M}^{n-2}$. If $\hat{c}\left[M_{\nu}\right]>0$ for all $\nu, 1 \leq \nu \leq l$, then

$$
\begin{aligned}
& <\Psi_{\hat{c}}(v), v_{i}>=c_{i} \quad \text { if } v \in s_{I} \cap \sigma_{i}, \\
& <\Psi_{\hat{c}}(v), v_{i}><c_{i} \quad \text { if } v \notin s_{I} \cap \sigma_{i},
\end{aligned}
$$

for any $i \in I$.

Proof. Proof will proceed by induction on $n$. By Lemma 3.4, $\left\langle\Psi_{\hat{c}}(v), v_{i}\right\rangle=c_{i}$ if $v \in \sigma_{i}$. Therefore it suffices to show that $\left\langle\Psi_{\hat{c}}(v), v_{i}\right\rangle\left\langle c_{i}\right.$ if $x \notin s_{I} \cap \sigma_{i}$. For $i \in I$, let $J \in \Sigma_{M}^{n-2}$ and $I^{\prime} \in \Sigma_{M}^{n-1}$ be defined by $\{i\} \cup J=I$ and $I \cap I^{\prime}=J$.

Consider first the case $n=2$. If $n=2, J=\{j\}$ and $s_{I}$ is divided into two parts $s_{I} \cap \sigma_{i}=\left|b_{i}, b_{I}\right|$ and $s_{I} \cap \sigma_{j}=\left|b_{j}, b_{I}\right|$. By Corollary 4.17, $<\Psi_{\hat{c}}\left(b_{j}\right), v_{i}><c_{i}$. Let $v \in s_{I} \cap \sigma_{j}=\left|b_{j}, b_{I}\right|$. Then $v$ is of the form

$$
v=t b_{j}+(1-t) b_{I}, 0 \leq t \leq 1 .
$$

Therefore $<\Psi_{\hat{c}}(v), v_{i}>=t \Psi_{\hat{c}}\left(b_{j}\right)+(1-t) c_{i}<c_{i}$ unless $t=0$.

Suppose $n>2 . M_{J}$ is contained in $M_{j}$ for each $j \in J$. By the inductive assumption applied on $M_{j},<\Psi_{\hat{c}}\left(x_{j}\right), v_{i}><c_{i}$ if $x_{j} \in s_{I-\{j\}}$ but $x_{j} \notin s_{I-\{j\}} \cap \sigma_{i}$. We also have $<\Psi_{\hat{c}}\left(b_{J}\right), v_{i}><c_{i}$ by Corollary 4.17. Since every $v \in s_{I}$ with $v \notin s_{I} \cap \sigma_{i}$ can be written as a linear combination with non-negative coefficients of the $x_{j}$ as above and $b_{J}$, the conclusion follows.

Let $L$ be a $T$-line bundle. Then there is an element $\delta \in H^{2}(B T ; \mathbf{Z})$ such that $L^{\prime}=$ $L \chi^{-\delta}$ comes from a $\bar{T}$-line bundle, and

$$
c_{1}^{T}(L)=c_{1}^{\bar{T}}\left(L^{\prime}\right)+\delta=\sum c_{i} \xi_{i}+\delta
$$

as remarked at the beginning of Section 4 . Moreover we have ind $D_{L}=\left(\operatorname{ind} D_{L^{\prime}}\right) \chi^{\delta}$.

TheOREM 4.19. Let $L$ be a positive line bundle over $M$. If we write

$$
\text { ind } D_{L}=\sum_{u \in H^{2}(B \bar{T} ; \mathbf{Z})} m(u) \chi^{u+\delta}
$$

then $m(u) \geq 0$. Moreover $m\left(u_{I}\right)>0$ for all $I \in \Sigma_{M}^{n-1}$, where $u_{I}=\Psi_{L^{\prime}}\left(b_{I}\right)$.

Proof. We may assume that $\delta=0$ from the start. By Theorem 2.9 and Theorem 3.11 ,

$$
m(u)=\bar{d}_{\hat{c}^{\prime}}(u) \text { for } u \in H^{2}(B \bar{T} ; \mathbf{Z}),
$$

where $\hat{c}^{\prime}=\sum\left(c_{i}+\frac{1}{2}\right) \xi_{i}$. However, it is easy to see that $\bar{d}_{\hat{c}^{\prime}}$ with $\hat{c}^{\prime}=\sum\left(c_{i}+r\right) \xi_{i}$ also satisfies the same identity (4.4) so far as $0<r<1$. We take $r$ small enough. Then, by Proposition 4.11, $\hat{c}^{\prime}$ is positive since $c_{1}^{\bar{T}}(L)$ is positive by assumption. Therefore

$$
m(u)=\bar{d}_{\hat{c}^{\prime}}(u) \geq 0
$$

by Proposition 4.7 .

Next we shall show $\bar{d}_{\hat{c}^{\prime}}\left(u_{I}\right)>0$ by induction on $n$. When $n=1, \Sigma_{M}^{0}$ consists of two points $b_{1}, b_{2}$ and $\left\langle u_{1}-u_{2}, v_{1}\right\rangle=c_{1}+c_{2}>0$, where $u_{i}=\Psi_{L}\left(b_{i}\right)$. Therefore $u_{1}$ and $u_{2}$ are contained in the unique bounded chamber for $\hat{c}^{\prime}$. Hence $\bar{d}_{\hat{c}^{\prime}}\left(u_{i}\right)>0$ for $i=1,2$. 
Suppose $n>1$. From the inductive assumption it follows that $\bar{d}_{\hat{c}^{\prime} \mid M_{i}}\left(u_{I}\right)>0$ on $F_{i}$ for any $i \in I$, where $F_{i}=\left\{u ; u \in H^{2}(B \bar{T} ; \mathbf{R}),<u, v_{i}>=c_{i}\right\}$ as before. Let $J \in \Sigma_{M}^{n-2}$ and $I^{\prime}$ be defined as in the proof of Lemma 4.18. Then $\left\langle u_{I}-u_{I^{\prime}}, v_{i}\right\rangle>0$ by Corollary 4.17. Take $\rho>0$ such that $\rho<u_{I}-u_{I^{\prime}}, v_{i}>=r$. Then $u_{I}+\rho\left(u_{I}-u_{I^{\prime}}\right)$ is in $F_{i}^{\prime}=\{u$; < $\left.u, v_{i}>=c_{i}+r\right\}$, and $\bar{d}_{\hat{c}^{\prime} \mid M_{i}}\left(u_{I}+\rho\left(u_{I}-u_{I^{\prime}}\right)\right)>0$ since $\bar{d}_{\hat{c}^{\prime} \mid M_{i}}$ is invariant by translation. Then the degree transition formula applied to the chamber containing $u_{I}$ and the wall containing $u_{I}+\rho\left(u_{I}-u_{I^{\prime}}\right)$ yields $\bar{d}_{\hat{c}^{\prime}}\left(u_{I}\right)>0$.

As an immediate corollary of Theorem 4.19 we obtain

Corollary 4.20. If $L$ is positive, then ind $D_{L}$ is a non-trivial honest $T$-module and $\operatorname{dim}$ ind $D_{L} \geq \# \Sigma_{M}^{n-1}$.

THEOREM 4.21. Let $L$ be a positive $T$-line bundle over $M$ and $K$ the canonical bundle of $M$. Then ind $D_{K \otimes L}$ is an honest $T$-module.

Proof. As in the proof of Theorem 4.19 we assume that $c_{1}^{T}(L)=\sum c_{i} \xi_{i}$ from the start. We write ind $D_{K \otimes L}=\sum_{H^{2}(B \bar{T} ; \mathbf{Z})} m(u) \chi^{u}$. Since $c_{1}^{T}(K)=-\sum \xi_{i}$ by Lemma 2.8,

$$
c_{1}^{T}(K \otimes L)=\sum\left(c_{i}-1\right) \xi_{i} \in \hat{H}_{\bar{T}}^{2}(M ; \mathbf{Z}) .
$$

Let $\epsilon>0$ be small enough. Put $r=1-\epsilon$ and $\hat{c}^{\prime}=c_{1}^{T}(K \otimes L)+r \sum \xi_{i}=\sum\left(c_{i}-\epsilon\right) \xi_{i}$. Then $\hat{c}^{\prime}$ is positive by Proposition 4.11 , and $m(u)=\bar{d}_{\hat{c}^{\prime}}(u) \geq 0$ for $u \in H^{2}(B T ; \mathbf{Z})$.

REMARK 4.22 . Theorem 4.21 can be thought of as an analogue of the Kodaira vanishing theorem. For details see [H2].

5. The case $T[M]=1$. In this section we show that some aspects of positive line bundles or more generally of positive classes $\hat{c}$ resemble those of ample line bundles in the theory of toric varieties. We keep the assumptions made on almost complex toric manifolds. Recall that the Todd genus $T[M]$ of $M$ equals the degree of the projection $\left|\Sigma_{M}\right| \rightarrow H_{2}(B \bar{T} ; \mathbf{R}) \backslash\{0\}$. The first observation is

LEMma 5.1. $T\left[M_{I}\right] \leq T[M]$ for all $I \in \Sigma_{M}^{k-1}, 1 \leq k \leq n$. In particular, if $T[M]=1$, then $T\left[M_{I}\right]=1$ for all $I$.

Proof. It is sufficient to show that $T\left[M_{i}\right] \leq T[M]$ for all $i \in \Sigma_{M}^{0}$. Take a generic circle subgroup $\bar{S} \subset \bar{T}$ such that $M^{\bar{S}}=M^{\bar{T}}$. Then we know that

$$
T[M]=\#\left\{p \in M^{\bar{S}} \text {; all } \bar{S} \text { weights at } p \text { are positive }\right\},
$$

see e.g. [H1]. Take $\bar{S}$ near $\bar{S}_{i}$ such that its projection on $\bar{T}_{i}=\bar{T} / \bar{S}_{i}$ is still generic. Then

$$
T\left[M_{i}\right]=\#\left\{p \in M_{i}^{\bar{S}} \text {; all } \bar{S} \text { weights of } M_{i} \text { at } p \text { are positive }\right\} .
$$

Let $v \in H_{2}(B \bar{T} ; \mathbf{Z})$ be the primitive element corresponding to the embedding $\bar{S} \subset \bar{T}$ and lying near $v_{i}$. If $p$ is in $M_{i}^{\bar{S}}$, then the $\bar{S}$ weights of $M$ at $p$ are the union of the $\bar{S}$ weights of $M_{i}$ at $p$ and $\left\langle\xi_{i}\right| p, v>$. But $\left\langle\xi_{i}\right| p, v>$ is close to $\left\langle\xi_{i} \mid p, v_{i}\right\rangle=1$. Hence $\left\langle\xi_{i} \mid p, v\right\rangle=1$. Therefore, if all the $\bar{S}$ weights of $M_{i}$ at $p$ are positive, then all the $\bar{S}$ weights of $M$ at $p$ are positive. This implies $T\left[M_{i}\right] \leq T[M]$. 
Hereafter we assume $T[M]=1$. We consider the following condition $(\mathrm{P})$ for $\hat{c} \in$ $\hat{H}_{\bar{T}}^{2}(M ; \mathbf{R})$ :

1. $\left\langle\Psi_{\hat{c}}\left(b_{I}\right), v_{i}\right\rangle \leq c_{i}$ for all $I \in \Sigma_{M}^{n-1}$ and $i \in \Sigma_{M}^{0}$.

2. The $u_{I}=\Psi_{\hat{c}}\left(u_{I}\right)$ are different to each other.

It is easy to see that the above condition (1) is equivalent to:

$\left(1^{\prime}\right)<\Psi_{\hat{c}}\left(b_{I}\right), v_{i}>\leq c_{i}$ for all $I \in \Sigma_{M}^{k-1}, 1 \leq k \leq n$ and $i \in \Sigma_{M}^{0}$.

Let $C S_{M}$ denote the cone over $S_{M}$ with the vertex $b_{0}$. We define a map $\tilde{\Psi}_{\hat{c}}: C S_{M} \rightarrow$ $H^{2}(B \bar{T} ; \mathbf{R})$ by

$$
\tilde{\Psi}_{\hat{c}}\left(t b_{0}+(1-t) v\right)=t u_{0}+(1-t) \Psi_{\hat{c}}(v)
$$

where $u_{0}=\frac{1}{\# \Sigma_{M}^{n-1}} \sum_{I \in \Sigma_{M}^{n-1}} u_{I}$, the barycenter of $\left\{u_{I} ; I \in \Sigma_{M}^{n-1}\right\}$. Put

$$
D_{\hat{c}}=\left\{u \in H^{2}(B \bar{T} ; \mathbf{R}) ;<u, v_{i}>\leq c_{i} \text { for all } i \in \Sigma_{M}^{0}\right\} .
$$

It is a convex set if it is not empty.

Lemma 5.2. The condition $(P)$ is equivalent to: $\operatorname{Im} \tilde{\Psi}_{\hat{c}}=\tilde{\Psi}_{\hat{c}}\left(C S_{M}\right)=D_{\hat{c}}$ and the $u_{I}$ are different to each other. In this case

$$
\bar{d}_{\hat{c}}(u)= \begin{cases}1, & u \in \text { interior } D_{\hat{c}} \\ 0, & u \in \text { outside } D_{\hat{c}}\end{cases}
$$

Proof. This is clear since $D_{\hat{c}}$ is the convex hull of $\left\{u_{I} ; I \in \Sigma_{M}^{n-1}\right\}$. The statement for $\bar{d}_{\hat{c}}$ follows readily by induction on $n$ and the degree transition formula.

Since $T[M]=1$, the complex $S_{M}$ (identified with $\left.\left|\Sigma_{M}\right|\right)$ is embedded in $H_{2}(B \bar{T} ; \mathbf{R})$ via $\pi$, and the vertex of the cone $C S_{M}$ can be taken at the origin $0 \in H_{2}(B \bar{T} ; \mathbf{R})$. Let $\angle s_{I}$ and $\angle \sigma_{i}$ denote the cone over $s_{I}$ and $\sigma_{i}$ respectively with the vertex at 0 . We define a function $\psi_{\hat{c}}: H_{2}(B \bar{T} ; \mathbf{R}) \rightarrow \mathbf{R}$ by

$$
\psi_{\hat{c}}(v)=<u_{I}, v>\text { if } v \in \angle s_{I}, I \in \Sigma_{M}^{n-1} .
$$

It is easy to see that $\psi_{\hat{c}}$ is well-defined.

LEMma 5.3. $\hat{c}$ satisfies the condition $(P)$ if and only if the function $\psi_{\hat{c}}$ is strictly lower convex.

This means that $\psi_{\hat{c}}$ is lower convex in the usual sense and $u_{I} \neq u_{I^{\prime}}$ for $I \neq I^{\prime}$. This is a familiar fact in the theory of toric varieties. We refer the reader to [O], Lemma 2.12. Here we remark that, in order to check the strict lower continuity of $\psi_{\hat{c}}$, it is sufficient to do it on each $\angle \sigma_{i}$ since it is linear on each $\angle s_{I}$ and the boundaries of $s_{I}$ are covered by $\left\{\sigma_{i}\right\}$.

TheOrem 5.4. Assume $T[M]=1$. Then the following condition are equivalent for $\hat{c} \in \hat{H}_{\bar{T}}^{2}(M ; \mathbf{R}):$

1. $\hat{c}$ is positive.

2. $\hat{c}\left[M_{J}\right]>0$ for all $J \in \Sigma_{M}^{n-2}$.

3. $\hat{c}$ satisfies the condition $(P)$.

Moreover, if $\hat{c}$ is positive then the image of $\tilde{\Psi}_{\hat{c}}$ coincides with $D_{\hat{c}}$. 
Proof. When $n=1$, it is easy to check the equivalence of the above two conditions as in the proof of Proposition 4.2.

Suppose $n>1$. The implication $(1) \Rightarrow(2)$ was proved in Proposition 4.7. The implication (3) $\Rightarrow(1)$ is easy in view of Lemma 5.2. Assume $\hat{c}\left[M_{J}\right]>0$ for all $J \in \Sigma_{M}^{n-2}$. Then Lemma 4.18 implies that $\psi_{\hat{c}}$ is strictly lower convex on each $\angle \sigma_{i}$. Therefore it is strictly lower convex on the whole $H_{2}(B \bar{T} ; \mathbf{R})$ as remarked after Lemma 5.3. Thus $\hat{c}$ satisfies the condition (P) by Lemma 5.3, and the image of $\tilde{\Psi}_{\hat{c}}$ coincides with $D_{\hat{c}}$ by Lemma 5.2.

As an immediate corollary of Theorem 5.4, Theorem 4.19 and Lemma 5.2 we obtain

Corollary 5.5. Assume $T[M]=1$. Then a $T$-line bundle $L$ is positive if and only if $c_{1}(L)\left[M_{J}\right]>0$ for all $J \in \Sigma_{M}^{n-2}$. In this case the non-zero multiplicities $m(u)$ are all equal to 1 .

REMARK 5.6. Corollary 5.5 is an analogue of the so-called toric Nakai criterion. See [O], Theorem 2.18; cf. also Remark 4.10.

\section{References}

[AB] M. Atiyah and R. Bott, The moment map and equivariant cohomology, Topology 23 (1984), 1-28

[D] T. Delzant, Hamiltoniens périodiques et image convexe de l'application moment, Bulletin Soc. Math. France 116 (1988), 315-339.

[F] W. Fulton, Introduction to Toric Varieties, Ann. of Math. Studies, 131, Princeton Univ. Press, 1993.

[GK] M. Grossberg and Y. Karshon, Equivariant index and the moment map for completely integrable torus actions, Adv. in Math. 133 (1998), 185-223.

[H1] A. Hattori, $S^{1}$-actions on unitary manifolds and quasi-ample line bundles, J. Fac. Sci. Univ. Tokyo 31 (1985), 433-486.

[H2] A. Hattori, Positive line bundle and index, Intern. J. Math. 7 (1996), 645-669.

[KT] Y. Karshon and S. Tolman, The moment map and line bundles over presymplectic manifolds, J. Diff. Geom. 38 (1993), 465-484.

[M] M. Masuda, Unitary toric manifolds, multi-fan and equivariant index, preprint (1997).

[O] T. Oda, Convex Bodies and Algebraic Geometry, An Intoduction to The Theory of Toric Varieties, Springer-Verlag, 1988. 